\title{
BMJ Open Can a tool developed for industry be used to assess fatigue risk in medical rotas? A pilot study of foundation doctors' rotas in a tertiary centre
}

\author{
Elspeth Cumber, ${ }^{1}$ Paul Robert Greig ${ }^{2}$
}

To cite: Cumber E, Greig PR. Can a tool developed for industry be used to assess fatigue risk in medical rotas? A pilot study of foundation doctors' rotas in a tertiary centre. BMJ Open 2019;9:e023470. doi:10.1136/ bmjopen-2018-023470

- Prepublication history for this paper is available online. To view these files, please visit the journal online (http://dx.doi. org/10.1136/bmjopen-2018023470).

Received 19 April 2018 Revised 18 December 2018 Accepted 8 January 2019

Check for updates

(C) Author(s) (or their employer(s)) 2019. Re-use permitted under CC BY-NC. No commercial re-use. See rights and permissions. Published by BMJ.

${ }^{1}$ Policy and Impact, Macmillan Cancer Support, London, UK

${ }^{2}$ Nuffield Department of Clinical Neurosciences, University of Oxford, Oxford, UK

Correspondence to Dr Elspeth Cumber; elspethcumber@gmail.com

\section{ABSTRACT}

Objectives To determine the feasibility and ease of using a pre-existing health and safety executive fatigue risk calculator to assess doctors' rotas.

Design Observational.

Setting A large tertiary-referral teaching hospital in the UK.

Participants 95 anonymised foundation years 1 and 2 rotas in General Medicine, General Surgery and Emergency Medicine covering a 4-month period. All rotas provided by rota coordinators were included and assessed.

Interventions Rotas were assessed for two indices: relative risk of fatigue-related errors compared with a '2-day, two-night, four-off' shift pattern and percentage chance of a high score on a standardised sleepiness scale. Primary and secondary outcome measures Fatigue index (percentage chance of a high score on a standardised sleepiness scale) and risk index (relative risk of fatigue-related errors compared with a '2-day, twonight, four-off' shift pattern) of all shifts on all rotas. Results Nearly half of all shifts demonstrated increased risk of fatigue-related errors and increased probability of high levels of sleepiness. There was significant interspeciality variation in both indices. These results are based on rotas as opposed to 'work as done' and are therefore likely to slightly universally underestimate actual fatigue risks.

Conclusions This preliminary study demonstrates that this tool can be used to compare rotas and guide rota design to minimise risk wherever possible. The calculator guidance clearly states there is no 'ideal risk' but that values should be minimised, and a maximum fatigue risk agreed which is deemed acceptable given the nature of work undertaken. This study is intended to demonstrate that fatigue can and should be considered during rota design. We do not suggest that it is used to hold either individuals or organisations to account as there is no evidence for it being used in this way. Further work should assess the practicality of designing medical rotas using this tool to minimise fatigue risk.

\section{INTRODUCTION}

Fatigue has been described as 'A physiological state of reduced mental or physical performance capability resulting from sleep loss or extended wakefulness, circadian phase, or
Strengths and limitations of this study

- Use of objective risk calculations offer new insights into fatigue management.

- This study uses a large sample size, covering different medical and surgical specialties.

- Fatigue risk indices are based on some assumptions on work intensity which, although plausible, are not based on prospectively gathered data.

- Health and safety executive risk calculator is based on work done in industrial settings rather than on healthcare staff.

workload (mental and/or physical activity) that can impair a crew member's alertness and ability to safely operate ... or perform safety related duties'.

This definition was originally written for the aviation industry, and in most safety critical industries, there are strict policies in relation to fatigue management. This is actively considered when planning staffing levels and working patterns, and redundancy is 'built-in' to rostering systems to enable the organisation to continue operating normally when staff are fatigued.

While regulation exists in medicine, working practices in hospital differ greatly from industry. This is in part related to differing resourcing available in a government-funded healthcare system such as the NHS, however culture also plays an important role. Long working hours and infrequent breaks are part of doctors' usual working life and has been for generations. In this, most doctors might be considered 'normalised deviants', because these practices are no longer viewed as hazardous. Indeed, most doctors would be surprised to hear the health and safety executive (HSE) specifically recommend against many normal patterns of medical shiftwork. ${ }^{3}$

Managing these risks is complicated, as medical services cannot easily be suspended 
or deferred, and relatively little work has been done on how to limit and manage short-term and long-term fatigue risks in healthcare. ${ }^{4}$ There have, however, been improvements in the years since the European Working Time Directive (EWTD) became part of UK law. The EWTD specifies maximum working periods and minimum rest requirements but does not specify how staff should be rostered for work. ${ }^{5}$ This is important, because not all work time is equivalent.

This is particularly true of 'out-of-hours' work. Nightshift workers accrue sleep debts, as sleep during daylight hours is reduced in both quantity and quality. ${ }^{6}$ Sequential night shifts compound risk ${ }^{78}$; over four nights, sleep debt is equivalent to having lost a whole night's sleep. This impairs performance to the same degree as a blood alcohol concentration above the UK drink-drive limit. ${ }^{9}$ One consequence of this is that trainees are at increased risk of being involved in road traffic collisions driving after night shifts. ${ }^{10}$ Fatigue also increases the likelihood of errors in the workplace, ${ }^{11}$ and self-assessment of fatigue-related performance impairment is poor. ${ }^{12}$ Circadian misalignment also carries long-term health risks for the worker. ${ }^{13}$

The nature of night shifts is also changing, with junior doctors caring for more patients of increasing complexity. Many hospitals have removed or reduced the provision of on-call bedrooms in the face of opposition from the British Medical association and Academy of Royal Colleges. ${ }^{14}$ This is unfortunate, because rest periods have been shown to reduce risk of workplace accidents, ${ }^{15}$ and this may be particularly relevant overnight.

Shift duration and rest periods are critical determinants of fatigue risk, but an often-overlooked contributor is how shifts are ordered on a rota. Training and guidance provided to medical rota writers on fatigue management is of variable quality and poorly standardised. Rotas are generally written to achieve regulatory compliance while accommodating leave requests, and fatigue risk is not necessarily afforded high priority.

This reflects that few tools exist in healthcare to assist rota-writers risk-assess their rota, but indices have been developed in other industries that might be applicable. The HSE developed a fatigue and risk index calculator, based on data collected in the rail industries, manufacturing and mining. This calculator uses estimates of work intensity, frequency of breaks and shift patterns to derive two indices. ${ }^{16}$

The first index describes the percentage chance of an individual experiencing high levels of sleepiness (scoring 8 or 9 on the Karolinska Sleepiness Scale, essentially a person who is fighting to stay awake). ${ }^{17}$ The second quotes the relative risk of making a fatigue-related error with reference to an 'idealised' working pattern of 12 hours shifts on a 2-day, two-night, 4 rest day schedule (note therefore, not risks compared with daytime working). These values allow comparison of duty schedules and can also aid the identification of areas of concern within an individual rota, before staff are required to work them.
In this study, the calculator has been used to assess foundation year 1 (FY1) and foundation year 2 (FY2) rotas. This tool has not previously been used to systematically analyse medical shift-work. This exploratory work intends to determine the ease with which this tool could be applied to medicine and will lay the foundation for future work considering its applicability in more detail.

\section{METHODS}

This study gained HRA approval (IRAS 208241), and was determined exempt from ethical review. This study has no patient or public involvement.

The study was carried out in a large tertiary-referral teaching hospital in the UK. Anonymised rotas covering a 4-month period were requested for all foundation year trainees working in acute General Medicine, acute General Surgery and Emergency Medicine.

The rotas provided were inputted into the HSE fatigue risk calculator. The calculator requires preset values for workload, mental demands, commute time and rest time. Given no personal data were collected, the authors used a best estimate for these values. Using definitions provided within the calculator, workload was set at 'moderately demanding, little spare capacity', representing the second-most demanding level of workload. Attention was set as 'requires continuous attention all or nearly all of the time'. Based on results of a recent survey, it was felt that this was a fair average across all the rotas. ${ }^{18}$ In a recent national survey of anaesthetists, ${ }^{10} 59.7 \%$ responded that their commute took in excess of $30 \mathrm{~min}$. This was therefore used as the commute-time estimate for calculations. With regard to breaks, it was assumed that doctors were taking breaks as advised in the 'New Deal' and 'EWTD', with 20 min break for each 6 hours worked. ${ }^{5}$ These values were set and remained constant for all rotas analysed.

Neither fatigue risk nor relative risk scores were normally distributed, as assessed by the Shapiro-Wilk test. Comparisons of continuous variables were made using non-parametric methods, such as the Kruskall-Wallis test. Proportional data were compared using $X^{2}$ tests. Two-sided $\mathrm{p}$-values are quoted for all comparisons.

\section{Patient and public involvement}

There was no patient or public involvement in this study. This study assessed the feasibility of a risk assessment tool in analysis of rotas, meaning patient or public involvement would not have been appropriate, with no intervention or direct impact on patients or the public.

\section{RESULTS}

\section{Demographic data}

These data covered both FY1 and FY2 doctors in medicine and surgery. No FY1 doctors are posted to the Emergency Department in this institution, so only FY2s data were available. These data represented the working pattern for 
Table 1 Number of rotas and total number of shifts for each specialty

\begin{tabular}{llc}
\hline & Staff & Total shifts \\
\hline Emergency medicine & 19 & 581 \\
General medicine (FY1) & 22 & 1836 \\
General medicine (FY2) & 16 & 1207 \\
General surgery (FY1) & 20 & 1630 \\
General surgery (FY2) & 18 & 1458 \\
Total & 95 & 6712 \\
\hline
\end{tabular}

nearly 100 members of staff, covering 6712 shifts. These data are summarised in table 1.

\section{Relative risk for fatigue-related errors}

Nearly half of all shifts analysed in the data set demonstrated increased risk of fatigue-related errors. Overall, 272 shifts $(4.1 \%)$ showed a relative risk greater than 1.5. The FY2 general medicine rota demonstrated the highest proportion of shifts with risk greater than $1(57.8 \%)$, followed by the medical FY1 rota $(50.1 \%)$. The Emergency Medicine rota had the lowest proportion of shifts with relative risk greater than $1(39.2 \%)$. These data are summarised in table 2 and figure 1 . The 'riskiest' shift in the data demonstrated a relative risk of 1.86 . Shifts in 14 of the 16 medical FY2 rota received this score, with the remaining two rotas scoring maximum values of 1.84 and 1.82 , respectively.

There was a significant association between the proportion of shifts with relative risk greater than 1 and specialty $(\mathrm{p}<0.001)$ as demonstrated by $\mathrm{X}^{2}$ testing. Kruskall-Wallis tests confirmed that relative risk indices varied significantly between specialties $(p<0.001)$.

\section{Fatigue risk indices}

More than half of all shifts analysed in the data set demonstrated increased probability of high levels of sleepiness. Overall, 862 shifts $(12.8 \%)$ showed a risk greater than $50 \%$. The FY2 general surgical rota demonstrated the highest proportion of shifts with risk greater than $10 \%$ $(62.8 \%)$, followed by the surgical FY1 rota $(58.4 \%)$. The General Medicine FY1 rota had the lowest proportion of shifts with risk greater than $10 \%(49.3 \%)$. These data are summarised in table 3 and figure 2. The 'riskiest'

\begin{tabular}{|c|c|c|c|c|}
\hline & \multicolumn{2}{|c|}{ Shifts risk $>1$} & \multirow{2}{*}{$\begin{array}{l}\text { Max } \\
\text { risk }\end{array}$} & \multirow{2}{*}{$\begin{array}{l}\text { Min } \\
\text { risk }\end{array}$} \\
\hline & $\mathbf{n}$ & $\%$ & & \\
\hline Emergency medicine & 228 & 39.24 & 1.63 & 0.82 \\
\hline General medicine (FY1) & 920 & 50.11 & 1.60 & 0.75 \\
\hline General medicine (FY2) & 698 & 57.83 & 1.86 & 0.74 \\
\hline General surgery (FY1) & 771 & 47.30 & 1.77 & 0.79 \\
\hline General surgery (FY2) & 712 & 48.83 & 1.59 & 0.78 \\
\hline Total & 3329 & 49.60 & 1.86 & 0.74 \\
\hline
\end{tabular}

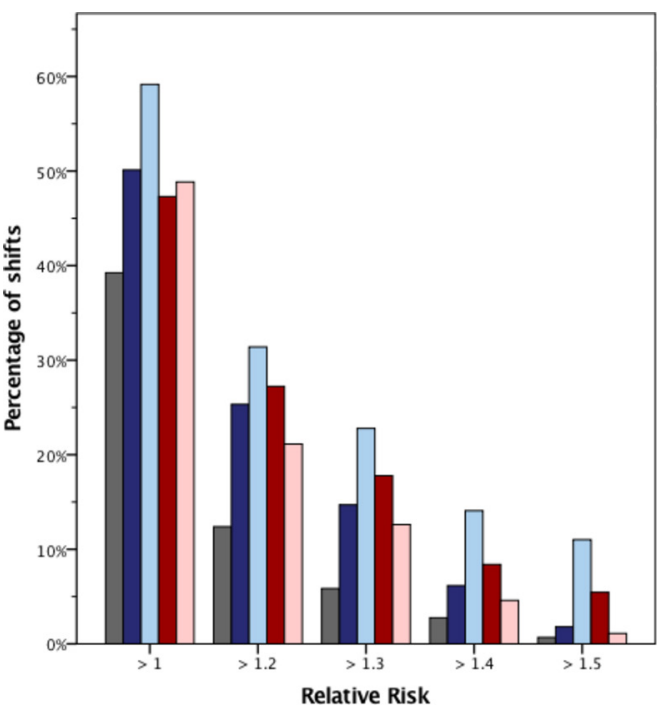

Specialty Emergency Medicine
Ceneral Medicine (FF1) Ceneral Medicine ( $\mathrm{FY} 1$
Ceneral Medicine (FY2) Ceneral Surgery (FY1)
General Surgery (FY2)

Figure 1 Percentage of shifts at differing levels of relative risk, analysed by specialty.

shift in the data demonstrated fatigue risks in excess of $70 \%$. Rotas in two of the five specialty areas had shifts that received scores greater than $66 \%$. Seventeen of 22 medical FY1, and all medical FY2 rotas incorporated at least one shift at this highest level or risk. The remaining five medical FY1 rotas each scored in excess of $64 \%$.

There was a significant association between the proportion of shifts with relative risk greater than $10 \%$ and specialty $(p<0.001)$ as demonstrated by $\mathrm{X}^{2}$ testing. Kruskall-Wallis tests confirmed that fatigue risk indices varied significantly between specialties $(\mathrm{p}<0.001)$.

\section{DISCUSSION}

Fatigue is undeniably complex and is affected by many factors within and beyond the workplace. Although the HSE calculator does not account for all possible factors contributing to fatigue, the simplicity with which it can be used makes it potentially valuable as a screening tool. Such tools can and should be used to begin to better mitigate the risk of fatigue-related errors; in the experience of the authors, fatigue management is not afforded high priority in rota design, possibly due to the complexity of the issue or the difficulty in quantifying the risks. While this tool cannot produce dichotomous 'acceptable' or

Table 3 Summary of fatigue risks, analysed by specialty

\begin{tabular}{lrrrrr} 
& \multicolumn{2}{c}{ Shifts risk $>\mathbf{1 0}$} & & Max & Min \\
\cline { 2 - 3 } & $\mathbf{n}$ & $\mathbf{\%}$ & risk & risk \\
\hline Emergency medicine & 335 & 57.66 & & 57.78 & 4.79 \\
General medicine (FY1) & 905 & 49.29 & 70.09 & 4.22 \\
General medicine (FY2) & 645 & 53.44 & 66.76 & 3.17 \\
General surgery (FY1) & 953 & 58.47 & 63.35 & 3.90 \\
General surgery (FY2) & 915 & 62.76 & 63.43 & 4.56 \\
Total & 3753 & 55.91 & 70.09 & 3.17 \\
\hline
\end{tabular}




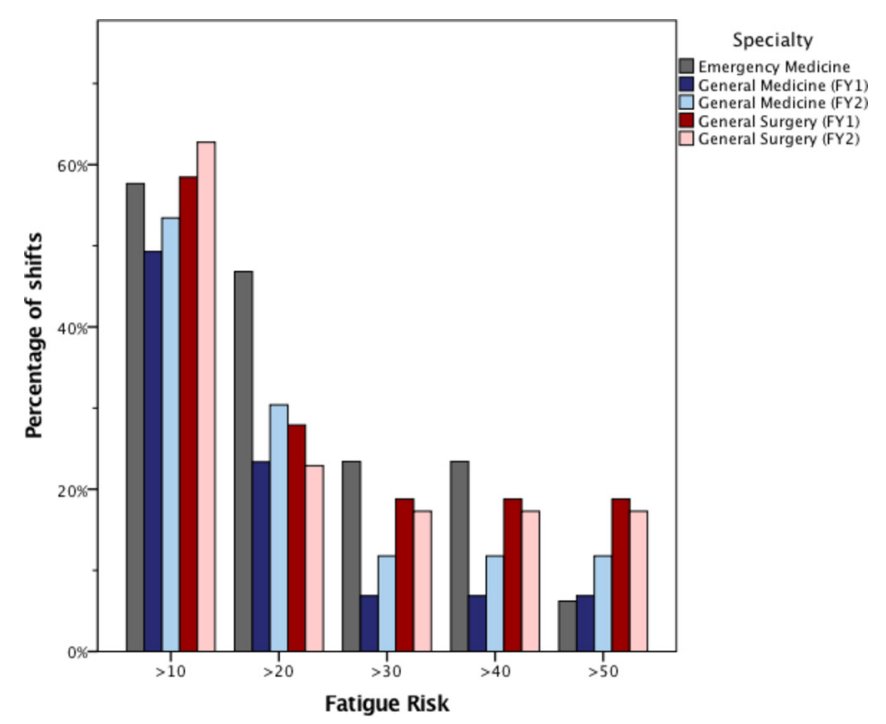

Figure 2 Percentage of shifts at differing levels of fatigue risk, analysed by specialty.

'unacceptable' value for a rota, it does at least offer a means of identifying high-risk clusters of shifts.

Current regulations stipulate strict rules around the duration of shifts and set minimum rest requirements, but offer little guidance about how shifts should be organised. The express purpose of regulation is to protect safety and health in the workplace, but the data presented here demonstrate clearly that even within the bounds of an EWTD-compliant rota, there may be significant variability in the likelihood of fatigue-related problems.

The Karolinska Sleepiness Scale is a nine-point Likert scale designed to provide a subjective assessment of an individual's level of drowsiness. Individuals scoring nine are described as 'extremely sleepy/fighting sleep'. Performance at this point is known to be impaired, but during more than half of the shifts in this data set staff were exposed to increased risk of this level of fatigue. That every medical FY2 shift had shift-clusters that yielded $60 \%$ chance of experiencing this level of fatigue is cause for concern. It is important to note that this relative risk is quoted against an idealised night-shift pattern, and not with reference to daytime working.

These hazards can put both patients and staff at risk ${ }^{910}$ : accidents and injuries are more frequent when staff are tired, and the consequences can be fatal. ${ }^{19}$ Rest periods are therefore essential in 'paying off' the accrued sleep debt, although surprisingly there is some evidence that the required 2 days of recovery after working nights specified by the EWTD and new junior doctor contract may not always be sufficient to fully recover. ${ }^{20}$

It is the authors' experience that hospital rota-writers receive little or no training in fatigue management, and the data presented here demonstrate the approach to these risks is not well standardised. The HSE make it clear that the calculator cannot be used to produce values to demonstrate a shift pattern is 'unacceptable', thus these decisions are the responsibility of staff managers, who must decide what level of risk is acceptable given the nature of work undertaken. With this in mind, there is no 'perfect' value to aim for, but the tool may be used to risk-assess rotas at the point of design. This would enable rota-writers to identify potentially challenging clusters of shifts, and explore the impact of potential revisions, before staff are expected to work them. There is no evidence that this is something which has ever been considered in the context of medical rotas previously, and this study is intended to highlight the potential applicability of this tool.

An additional point was noted during the analysis of these data. With the exception of the emergency medicine rota, the working patterns in these documents were 'rolling' in nature. This meant that the shift-patterns were fixed across the rota period, and as new staff joined the team they were inserted into a vacant 'slot'. It was observed that, depending on where in the rota an individual started, the number of days worked over the period was highly variable. In some instances, there were as many as 11 working days difference over a 4-month period between individuals ostensibly working the same job. Writing rolling rotas is convenient from an organisational perspective, but it appears to carry the potential of inequity, and may be an additional source of tension between staff members in the workplace.

One of the limitations of this study is that it captures work as described, not work as done. We cannot quantify the actual hours worked by doctors staffing these rotas. It is not, however, implausible to suggest that these data may even be 'best case' and that many doctors, particularly at the start of their posts, work many additional hours. ${ }^{18}$ It is also commonly argued that these tools are not validated for use in healthcare. While it is true that the HSE risk index was originally developed for use in the rail industry, that has not stopped it being used extensively in industry and featuring prominently in, for example, air-accident investigations. The underlying data were generated using analysis of manufacturing workers, transport employees and miners, ${ }^{15}$ all of whom have different workplaces and responsibilities. One must also question why we should consider healthcare to be different, as our staff share the same physiology as workers in other fields and are driven by the same circadian rhythms. To suggest otherwise risks perpetuating the myth that Farquhar has described as 'healthcare heroism'. 21

A final limitation is that it is not clear how different specialities might be differentially affected by fatigue. Mental and physical fatigue accrue and recover somewhat differently, thus fatigue level may vary between areas of practice. The foundation-trainee population across a single hospital is a relatively homogeneous population, which reduces this problem in the current study, but would be a factor to consider if deploying this tool across a more varied group. It must be recognised that the intensity of work throughout a shift can be significantly varied across specialities, particularly in grades higher than FY2. 
Also, the risks associated with fatigue as experienced by the patient will vary in certain situations. When comparing an FY doctor to a consultant with similar fatigue levels, the risks associated with fatigue may be higher for the consultant in that the more senior clinician is responsible for decisions that carry greater consequences. However, clinician experience, age and the specific task or decision being undertaken will all affect the impact of fatigue, making the correlation with risk less clear. Risks are also sensitive to the safety processes in each workplace; robust error-trapping processes will reduce risk for a given level of fatigue. Fatigue levels and fatigue risk are thus closely linked, but not interchangeable concepts.

The results of this study demonstrate that how shifts are ordered is crucially important in determining fatigue risk. This should be considered at least as important as the total number of hours worked. The values output from this tool may not give a perfect measure of workplace fatigue, but the overall pattern of high and low risk periods in the rota is useful information. We have no better means of measurement at present, and at a very minimum, it could be used productively as a 'screening test'. The tool can indicate where a rostering pattern might be at higher than average risk, or where shift patterns deviate from what other industries would consider best (or even usual) practice. This offers an opportunity to experiment with different patterns before deployment.

The methods used here can be used to assess any specialty and grade of staff, in any trust. The tool is already used in critical incident investigation by the Air Accident Investigation Branch. In 2002, David Gaba suggested that 'if the same [fatigue] analysis were applied to accidents involving the care of patients in teaching hospitals, fatigue on the part of clinicians would almost always be cited as a contributing factor, ${ }^{22}$ It is likely that a tool such as this may be useful when conducting investigations such as root cause analyses in screening working patterns for additional latent risk factors.

This calculator, freely available through the HSE, is simple to use, and takes little training to understand. It therefore gives rota-writers, staff and healthcare managers a pragmatic method to quantify fatigue risks. These tools allow rota-writers to experiment with different patterns and have some objective method of assessing the impact of proposed changes.

These data suggest that adhering to simple rules can improve fatigue management within existing staffing levels, with no additional costs. Rota-writers should look to industrial recommendations for guidance to minimise risk. For example, it is easier for people to lengthen their day rather than shorten it, ${ }^{23}$ so moving shifts 'clockwise' (days, to late-, to night-shifts) over a given period is considered best practice. ${ }^{24}$ These adjustments require leadership, but should be relatively easy to implement, even within current resourcing. While we cannot change the 24 hours demand for healthcare, we should acknowledge that the work carries significant risk, and take whatever steps are possible to alleviate this. We believe that this tool offers an opportunity screen rotas for periods of high fatigue risk, and welcome further work focused more specifically on individual circumstances which is specific to healthcare. It would be particularly valuable to consider how fatigue differentially affects different specialties.

Contributors Data collection was conducted by EC. Analysis and authorship of the manuscript was conducted jointly by both EC and PRG. There was no patient or public involvement.

Funding The authors have not declared a specific grant for this research from any funding agency in the public, commercial or not-for-profit sectors.

Competing interests None declared.

Patient consent for publication Not required.

Ethics approval The protocol for this study gained HRA approval (IRAS 208241). The protocol was reviewed by NRES and it was determined ethical review was not required.

Provenance and peer review Not commissioned; externally peer reviewed.

Data sharing statement Data available by request from the corresponding author.

Open access This is an open access article distributed in accordance with the Creative Commons Attribution Non Commercial (CC BY-NC 4.0) license, which permits others to distribute, remix, adapt, build upon this work non-commercially, and license their derivative works on different terms, provided the original work is properly cited, appropriate credit is given, any changes made indicated, and the use is non-commercial. See: http://creativecommons.org/licenses/by-nc/4.0/.

\section{REFERENCES}

1. International civil aviation authority. Doc 9966 Manual for the Oversight of Fatigue Management Approaches. 2016 http://www. icao.int/safety/fatiguemanagement/FRMS\%20Tools/Doc\%209966. FRMS.2016\%20Edition.en.pdf

2. NHS Employers. Unsocial hours and night working. 2018. Accessed http://www.nhsemployers.org/ /media/Employers/Publications/ Unsocial\%20hours\%20and\%20night\%20working.pdf\#

3. Great Britain. Health and Safety Executive. Managing shift work: health and safety guidance: HSE Books, 2006:36.

4. Morrison I, Flower D, Hurley J, et al. Working the night shift: a necessary time for training or a risk to health and safety? $J R$ Coll Physicians Edinb 2013;43:230-5.

5. BMA. European Working Time Directive for Junior Doctors [Internet]. bma.org.uk. 2014 http://www.bma.org.uk/support-at-work/ewtd (cited 5 Apr 2016).

6. Johnson WB, Mason F, Hall S, et al. Evaluation of aviation maintenance working environments, fatigue, and human performance executive summary. 2001.

7. Folkard S, Lombardi DA, Tucker PT. Shiftwork: safety, sleepiness and sleep. Ind Health 2005;43:20-3.

8. Lerman SE, Eskin E, Flower DJ, et al. Fatigue risk management in the workplace. J Occup Environ Med 2012;54:231-58.

9. Dawson D, Reid K. Fatigue, alcohol and performance impairment. Nature 1997;388:235.

10. McClelland L, Holland J, Lomas JP, et al. A national survey of the effects of fatigue on trainees in anaesthesia in the UK. Anaesthesia 2017;72:1069-77.

11. Philibert I. Sleep loss and performance in residents and nonphysicians: a meta-analytic examination. Sleep 2005;28:1392-402.

12. Philip $P$, Sagaspe $P$, Taillard J, et al. Fatigue, sleep restriction, and performance in automobile drivers: a controlled study in a natural environment. Sleep 2003;26:277-80.

13. Shift work and sleep: optimizing health, safety, and performance. $J$ Occup Environ Med 2011;53:S1-S10.

14. Joint JDC/AoMRC Trainees' Committee position statement on oncall rooms. 2018. Accessed http://www.asit.org/assets/documents/ JDC_AoMRC_oncallrooms.pdf

15. Folkard S, Lombardi DA. Modeling the impact of the components of long work hours on injuries and "accidents.". Am J Ind Med 2006;49:953-63.

16. Folkard S, Limited A, Spencer MB, et al. The development of a fatigue / risk index for shiftworkers Prepared by QinetiQ Centre for Human Sciences The development of a fatigue / risk index for shiftworkers. 2006. 
17. Kaida K, Takahashi M, Akerstedt T, et al. Validation of the Karolinska sleepiness scale against performance and EEG variables. Clin Neurophysiol 2006;117:1574-81.

18. General Medical Council. 2017 national training surveys summary report: initial results on doctors' training and progression. 2017 https://www.gmc-uk.org/2017_national_training_surveys_summary report_initial_results_on_doctors_training_and_progression.pdf_ 71003116.pdf

19. Doctors "fall asleep driving" after punishing night shifts, the BBC learns. - BBC News [Internet]. http://www.bbc.co.uk/news/av/ uk-england-berkshire-38716140/doctors-fall-asleep-driving-afterpunishing-night-shifts-the-bbc-learns (cited 2017 Nov 27).
20. Borbély AA. A two process model of sleep regulation. Hum Neurobiol 1982;1:195-204.

21. Farquhar M. For nature cannot be fooled. Why we need to talk about fatigue. Anaesthesia 2017;72:1055-8.

22. Gaba DM, Howard SK. Patient safety: fatigue among clinicians and the safety of patients. N Engl J Med 2002;347:1249-55.

23. Recht LD, Lew RA, Schwartz WJ. Baseball teams beaten by jet lag. Nature 1995;377:583.

24. Health and safety executive. HSE Human Factors Briefing Note No 10 - Fatigue. http://www.hse.gov.uk/humanfactors/topics/ 10fatigue.pdf 